\title{
Crónica
}

\section{OBJ EIIVOS SANITARIOS PARA LA DÉCADA 2000-2010. EVALUACIÓN A MITAD DEL PERÍODO}

Fragmento en relación a salud de la mujer del documento: "Objetivos Sanitarios para la Década 2000- 2010. Evaluación a mitad del período". Documento completo en: http://www.minsal.cl/ici/destacados/ Evaluacion_de_los_Objetivos_Sanitarios_de_Impacto_a_mitad_de_periodo.pdf

\section{Salud de la mujer}

\section{a. Objetivos con avance}

La tasa de mortalidad materna a 2004 fue de 1,7 por 10.000 nacidos vivos, lo que implica una reducción del 26\% en relación a 1999 (2,3 x $10.000 \mathrm{NV}$ ). La meta al 2010 es llegar a 1,2 por 10.000 NV (Gráfico I. 3). La tasa de mortalidad materna relacionada con aborto en 2003 y 2004 fue de 0,2 por 10.000 nacidos vivos, mostrando estabilidad en los últimos años. En esta mejoría han influido el mayor número de controles prenatales y de regulación de fecundidad. Asimismo, el control preconcepcional realizado por matrona en APS (atención primaria de salud), aumentó de 33.820 en el año 2001, a 47.621 en el año 2003. En lo referente a los controles de regulación de fecundidad cubren al $40,3 \%$ de las 2.670 .004 mujeres en edad fértil inscritas en establecimientos de atención primaria el año 2004 y han continuado creciendo; entre Diciembre del año 2003 y Junio del 2005, la población de mujeres bajo control en regulación de fecundidad en el sector público aumentó en un $4,3 \%$. Respecto al tipo de método utilizado, aumentó el uso de métodos hormonales en un $13,6 \%$ y el de preservativos en un $9 \%$; casi se duplicó el número de mujeres que utilizan anticonceptivos inyectables de uso mensual (de 8.464 usuarias a 16.070) y disminuyó el número de hombres que recibió preservativos (de 9.125 a 7.176). A pesar de que no se estableció una meta cuantitativa a 2010 , se observa que la tasa de mortalidad por complicaciones obstétricas se ha reducido en $47 \%$ en relación con la tasa observa- da en 1999, en 2003 fue de 0,28 por 10.000 NV. La tasa de fecundidad en mujeres de 15 a 19 años bajó de 65,4 por mil mujeres de este grupo de edad en 1999 a 49 en 2004, lo que implica un avance de $84 \%$ de la meta planteada a 2010 (46 por mil mujeres de 15 a 19 años). Dado el contexto legal (aborto penalizado), y la falta de datos correspondiente a la población beneficiaria del sistema privado de salud, no se dispone de información acerca del número real de adolescentes que inician un embarazo cada año. De acuerdo a información del sistema público de salud, el porcentaje de embarazadas menores de 20 años bajo control en el SNSS, se ha mantenido estable entre los años $2002(21,1 \%)$ y $2004(21,7 \%)$ (Fuente: DEIS MINSAL). Si bien se observa una leve disminución de los nacidos vivos hijos de madres adolescentes entre 2000 y 2004, no es posible atribuir esta disminución a un mayor acceso de la población adolescente a los servicios que le ofrece el SNSS (consejería, uso de método anticonceptivo), ya que entre los años 2003 y 2005 se redujo la propor-

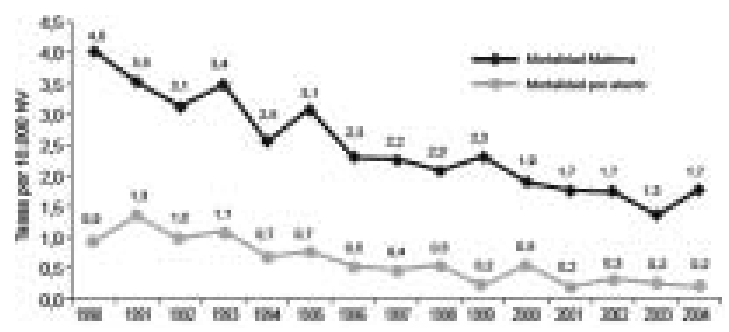

Gráfico I. 3. Mortalidad materna y por aborto. Chile, 19902004. 
ción de adolescentes en Control de Regulación de la Fecundidad, pasando de $10,7 \%$ a $10,1 \%$, respecto a la población mayor de 20 años. La calidad de la atención obstétrica tiene un avance discreto; se elaboró la Guía Perinatal y se espera la incorporación de analgesia obstétrica a las garantías explícitas en salud a contar de mediados del 2007.

\section{SOCHOG Y SOCIEDAD DE GINEC OLOGÍA Y OBSTEIRICIA DE CANADÁ FIRMARON ACUERDO DE MUTUA COLABORACIÓN}

En el marco de la reunión de directorio del martes 12 de diciembre de 2006, la Sociedad Chilena de Obstetricia y Ginecología (SOCHOG) firmó un acuerdo de mutua cooperación con la Sociedad Canadiense de Obstetricia y Ginecología (SOGC). Esta gestión implica un reconocimiento internacional al desarrollo de nuestra especialidad en el país.

En representación de Canadá, firmó el acuerdo el Dr Donald Davis, Presidente de SOGC, quien hace muy pocas semanas fue uno de los principales impulsores de la reelección de Chile en el Executive Board de FIGO, durante el XVIII Congreso de esa Federación, realizado en noviembre en Malasia. La entidad canadiense optó por asociarse con SOCHOG, bajo la denominación de Partner Chips, con el objetivo de realizar en forma conjunta tareas que la SOGC debe ejecutar para colaborar en el desarrollo de la especialidad en el mundo.

Cabe recordar, que los países desarrollados se comprometieron con la ONU a invertir un 0,7\% de su PIB para ir en ayuda de países en vía de desarrollo. Dicha ayuda se materializa a través de programas concretos que cuentan con estrictos procesos de evaluación. Los principales horizontes definidos, eran luchar contra el hambre y la mortalidad materna. En el caso de Canadá el compromiso fue aportar con $0,4 \%$, y lo harían a través de instituciones prestigiosas. En el último tiempo han focalizado sus esfuerzos en países como Uganda, Haití y Guatemala, y han logrado muchos avances. Sólo en este último país centroamericano, lograron bajar la mortalidad de alrededor 400 por cada cien mil, a la décima parte.

EI paso siguiente de SOGC es ir en ayuda de países latinoamericanos con altos índices de mortalidad materna. Sin embargo, no cuentan con muchos profesionales que puedan viajar hasta esas naciones, y además existe la barrera del idioma. Por eso les pareció conveniente una alianza con un país sudamericano cercano, que cuente con un buen nivel de la especialidad para ser un socio estratégico en esta tarea. SOCHOG fue elegida en reconocimiento a su buen trabajo, y a las bajas cifras de mortalidad materna en Chile.

Este programa de ayuda, ejecutado por países desarrollados en pro de los más pobres se denomina ALARM. En forma paralela a él, también llevan a cabo el programa MORE, que consiste en ayudar a elevar la calidad obstétrica en países en desarrollo y subdesarrollo. La intención de SOCHOG es acceder a este último programa, ya que si bien tenemos muy buen nivel en el área obstétrica, el alto índice de cesáreas revela que aún tenemos un asunto por resolver.

Trabajar en forma conjunta en ambos programas obligará a ambas agrupaciones ha reunirse semestralmente, para compartir experiencias y traspasar conocimientos. El acuerdo, que no limita la autonomía de las instituciones que la suscribieron, es válido por cinco años, y puede ser extendido por decisión mutua.

Este acuerdo, finalmente, da un respaldo aún mayor al proyecto de generar un capítulo chileno del American College of Obstetric and Gynecology, el cual tomó mayor fuerza a partir de la reelección de SOCHOG en el Executive Board de FIGO, momento en el cual la institución norteamericana invitó a la entidad nacional a formar esa representación. Por ello, el Dr. Ralph Hale, vicepresidente administrativo de ACOG, se reunirá en Chile en enero de 2007 con el directorio de SOCHOG a establecer las pautas necesarias para concretar este proyecto.

\section{Dr. Eghon Guzmán B. Presidente.}

Sociedad Chilena de Obstetricia y Ginecología. 


\section{SOCHOG FUE REELEGIDA MIEMBRO DEL EXECUTIVE BOARD DE FIGO}

En el marco del XVIII Congreso de la Federación Internacional de Ginecología y Obstetricia (FIGO), realizado entre el 5 y 10 de noviembre de 2006 en Malasia, la Sociedad Chilena de Obstetricia y Ginecología (SOCHOG) fue reelegida como miembro integrante de los 24 países que conforman el Executive Board de FIGO, a partir del cual se definen las políticas y los planes del organismo internacional que agrupa a entidades representantes de 110 países.

Se agradeció particularmente el apoyo brindado por Canadá, Estados Unidos, Guatemala, China y Japón, quienes dieron su voto a la entidad chilena. Conjuntamente, se destacó el rol del Dr André Lalonde, vice-presidente de Society of Obstetricians and Gynecologists of Canadá (SOGC), quien fue uno de los principales impulsores de la presencia de Chile en este comité.

En el circuito mundial, Chile cuenta con un gran prestigio en el área de la obstetricia y ginecología. La reelección implica una enorme responsabilidad y trabajo. Desde allí, continuaremos influ- yendo directamente en las políticas mundiales orientadas a proteger la salud de la mujer. Por otra parte, durante el congreso mundial la ginecóloga canadiense Dorothy Shaw asumió el cargo de presidenta de la institución, convirtiéndose en la primera mujer en ejercer esta función. La profesional estará en el cargo hasta el 2009.

A partir de la reelección de SOCHOG en el Executive Board, el Colegio Americano de Obstetricia y Ginecología (ACOG) invitó a la entidad nacional a formar un capítulo chileno del organismo estadounidense. El Dr Ralph Hale, vicepresidente administrativo de ACOG, se reunirá en Chile en enero de 2007 con el Directorio de SOCHOG a establecer las pautas necesarias para lograr ese objetivo en nuestro país.

\section{Dr. Eghon GuZMÁn B. \\ Presidente.}

Sociedad Chilena de Obstetricia y Ginecología. 